\title{
Characterization of Dynamic Behaviors in a Hexapod Robot
}

Haldun Komsuoglu and Anirudha Majumdar and Yasemin Ozkan Aydin and Daniel E. Koditschek

\begin{abstract}
This paper investigates the relationship between energetic efficiency and the dynamical structure of a legged robot's gait. We present an experimental data set collected from an untethered dynamic hexapod, EduBot [1] (a RHex-class [2] machine), operating in four distinct manually selected gaits. We study the robot's single tripod stance dynamics of the robot which are identified by a purely jointspace-driven estimation method introduced in this paper. Our results establish a strong relationship between energetic efficiency (simultaneous reduction in power consumption and increase in speed) and the dynamical structure of an alternating tripod gait as measured by its fidelity to the SLIP mechanics - a dynamical pattern exhibiting characteristic exchanges of kinetic and spring-like potential energy [3]. We conclude that gaits that are dynamic in this manner give rise to better utilization of energy for the purposes of locomotion.
\end{abstract}

\section{Introduction}

Energetics of locomotion has been a major topic for scientific discussions [4-8] and remains to be an active research area for both biologists $[9,10]$ and engineers [11-15]. The need for practical mobility in unstructured environments

Haldun Komsuoglu

University of Pennsylvania, Philadelphia, USA, e-mail: haldunk@seas .upenn.edu

Anirudha Majumdar

University of Pennsylvania, Philadelphia, USA, e-mail: anirudha@seas .upenn.edu

Yasemin Ozkan Aydin

Middle East Technical University, Ankara, Turkey, e-mail: yasemino@eee.metu.edu.tr

Daniel E. Koditschek

University of Pennsylvania, Philadelphia, USA, e-mail: kod@seas .upenn.edu 
motivates our interest in legged locomotion. This paper presents experimental data suggesting a strong correlation between energetic efficiency and dynamic characteristics of legged locomotion.

Since its introduction specific resistance (SR) [4] has been the de-facto measure of locomotive competency. Being a dimensionless quantity, specific resistance allows us to evaluate and compare a wide spectrum of systems both of biological [16] and artificial [11,15, 17-20] origin from energetic efficiency perspective. In prior work researchers successfully utilized specific resistance [4] and its variants as objective functions to empirically tune [15] behavioral controllers in legged platforms. Unfortunately, specific resistance, treating the system as a black box, offers very little concrete design insight for either hardware or algorithms.

Roboticists in partnership with biologists have been seeking this missing design guidance in nature [21,22]. A key observation of this work is that animals of different size and morphology [28] minimize the average work required [27] by performing "proper" exchanges between different forms of kinetic energy, internal spring potential and gravitational potential through the use of passive body parts as conduits for these transfers [23-26]. A direct consequence of this observation is the emergence of the spring-loaded inverted pendulum (SLIP) model [3] as a template [39] to characterize the center of mass $(\mathrm{COM})$ behavior in dynamic ${ }^{1}$ animal locomotion.

Inspired by the descriptive power of the SLIP template for high performance animal locomotion, various robotic systems have been developed based on its blueprint [13,30-32]. Among these platforms biologically inspired power autonomous RHex-class robots [2] stand out because of 1) their ability to negotiate previously unaccessible terrains untethered [33]; 2) their wide behavioral repertoire; and 3) the volume of studies they have enabled [1,20,34-38]. Furthermore, while their morphology and actuation scheme diverge significantly from that of the SLIP model, RHex-class robots can passively anchor [39] the SLIP dynamics in properly tuned gaits [40]. This very observation in conjunction with SLIP's desirable characteristics and its ubiquitous appearance in biological systems have led to the speculation that embedding [39] SLIP dynamics in RHex behaviors (actively [41] and/or passively [40]) can lead to efficient use of energy in RHex-class platforms, and more importantly, offer constructive design ideas to the developers.

In this paper we empirically demonstrate a strong relationship between energetic efficiency and the emergence of SLIP behavior in a RHex-class machine. We define the "SLIP-measure" - a dimensionless scalar value evaluating the quality of dynamical activity - as the similarity between the trajectories of physical COM and the best fitting SLIP template starting from the same initial condition. Our results suggest that the energetic performance of a gait (as captured by the specific resistance) and the nature of its dynamical structure (as captured by the SLIP-measure) are (perhaps monotonically)

\footnotetext{
${ }^{1}$ We define dynamic behavior as those operations exhibiting significant exchange between kinetic and potential energy [29].
} 
related. This model based measure offers a more concrete gauge of specific characterization of dynamical activity in comparison to previous work $[42,43]$ which treats the system and its behavior as a black box.

The outline of this paper is as follows. Section 2 provides a detailed description of the experimental setup that was employed for data collection. Section 3 presents the post-processing pipeline. The system identification analysis and its results are presented in Section 4. We discuss the relationship between the behavioral energetics and the level of dynamism in Section 5 . Final remarks and extensions to be studied in future work are presented in Section 6.

\section{Experimental Setup}

This section describes our experimental setup and data cleanup method. This architecture - an improved version of the setup in [37] — facilitates the necessary systematic data collection in controlled environmental conditions.

EduBot (Figure 1(a)), whose variants have successfully served in a wide range of experimental robotics studies [1,37,38,44,45] and in classrooms [46], is a small form-factor legged mobility system that adopts the successful RHex morphology (Figure 1(b)) and task-level open-loop locomotion strategy [2].

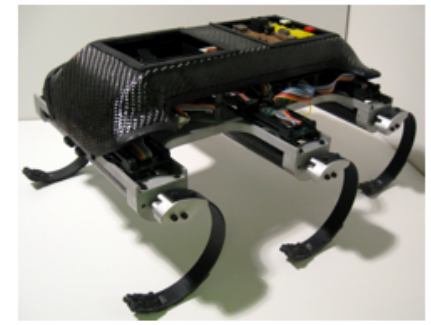

(a)

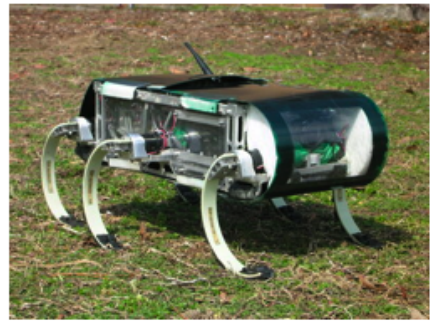

(b)

Fig. 1 (a) EduBot platform (b) RHex platform [2].

The platform weights $3.31 \mathrm{~kg}$. Its rigid rectangular body is supported and propelled by six half-circle shaped compliant legs each driven by an independent hip servo - an 11W brushed DC motor managed by an embedded motor controller that monitors and regulates the states of motor (its output shaft position and speed) at $1 \mathrm{KHz}$ update rate within hard real-time constraints. The robot is powered by an onboard 4-cell $1320 \mathrm{mAh} 20 \mathrm{C}$ Li-Poly battery whose output current and terminal voltage are monitored to assess total power consumption of the platform. The higher level behavioral control and proprioceptive sensory recording are performed by an on-board PC104 
stack powered by a Pentium class CPU running at 266MHz. A GUI application running on an external computer issues task level control commands and collects sensory data.

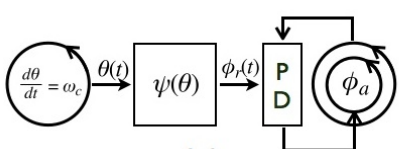

(a)

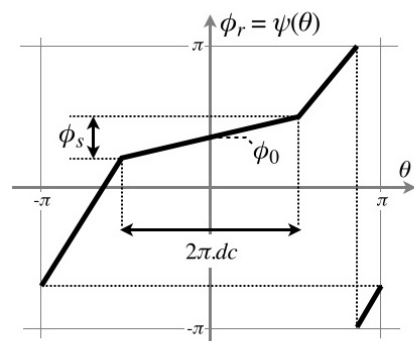

(b)

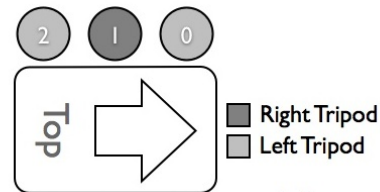

(c)



(d)

Fig. 2 (a) Two tripod leg groupings: the left tripod (front and rear left side legs and the middle right side leg); and the right tripod (front and rear right side legs and the middle left side leg) (b) The Buehler two speed profile is defined by three parameters: sweep angle, $\phi_{s}$; duty cycle, dc; and leg offset, $\phi_{0}$. In our implementation, PD gains, $k_{p}$ and $k_{d}$ are represented in a normalized coordinate system with values in $[-1,1)$ relating the normalized hip position, $\bar{\phi}:=\phi / \pi$, and normalized speed, $\overline{\dot{\phi}}:=\dot{\phi} /\left(10^{-3} \pi\right)$, to normalized terminal voltage, $\bar{V}:=V / 14.8$.

Our study uses the tripod gait controller [2] which implements a 6parameter family of behaviors that covers a wide range of locomotive characteristics [15,33]. We identified four tripod gaits, as listed in Table 1. These gaits were selected based on their apparent dynamic qualities coarsely representative of the range of dynamical activity that the robot can realize.

Table 1 The Behavioral Parameters for the Test Gaits (refer to Figure 2 for the behavioral interpretation of these parameters)

\begin{tabular}{|l|c|l|l|l|l|}
\hline \hline & units & Running & Jogging & Pace & Walk \\
\hline$w_{c}$ & $\mathrm{rad} / \mathrm{sec}$ & 19.0 & 17.4 & 10 & 6.0 \\
\hline$\phi_{s}$ & $\mathrm{rad}$ & 0.75 & 1.12 & 1.0 & 1.2 \\
\hline$\phi_{0}$ & $\mathrm{rad}$ & 0.35 & 0.2 & 0.2 & 0.0 \\
\hline $\mathrm{dc}$ & $\mathrm{N} / \mathrm{A}$ & 0.3 & 0.45 & 0.6 & 0.7 \\
\hline$k_{p}$ & norm & 0.56 & 0.56 & 0.7 & 0.7 \\
\hline$k_{d}$ & norm & 0.03 & 0.03 & 0.0 & 0.0 \\
\hline
\end{tabular}


The robot collects and transmits proprioceptive sensory measurements at $100 \mathrm{~Hz}$. This data stream consists of (1) time stamps for sensor sampling events; (2) measured actual hip positions, $\phi_{a}$, and speeds, $\dot{\phi}_{a} ;(3)$ controller generated target hip position, $\phi_{r}$, and speed, $\dot{\phi}_{r}$; and (4) the battery output current, $i_{b}$, and voltage, $v_{b}$. The active tripod gait parameters are also appended to the log file.

The Vicon system, shown in Figure 3, is a professional-grade ground truth motion tracking setup that is capable of simultaneously tracking multiple passive reflective markers in its workspace at $120 \mathrm{~Hz}$. Using the positions of tracked markers and their organization on robot's chassis the Vicon system computes 6-dof configuration of the rigid robot body, $\mathbf{c}^{\mathcal{V}}$, consisting of the COM position, $\left[b_{x} b_{y} b_{z}\right]^{T}$ and body orientation, $[\beta \alpha \rho]^{T}$, with respect to Vicon coordinate system, $\mathcal{V}:=\mathbb{R}^{3} \times S O(3)$, that is rigidly attached to the World Coordinates, $\mathcal{W}$. The roll, $\rho$, pitch, $\alpha$, and yaw, $\beta$, are computed using the Rodrigues' rotation formula. Each data point is also tagged by a frame index which serves as a time stamp.



Fig. 3 The Vicon system consists of six digital high speed cameras with back light each viewing from a different angle the $4 \times 3 \times 1.5 \mathrm{~m}$ workspace where the robot runs. In the figure blue tetrahedra represents the individual camera view space. The robot body configuration tracking is only accurate over the permissible domain, $\mathcal{P}$, measuring $2 \times 1 \times 1 \mathrm{~m}$ - a subset of the workspace. The floor is covered with high friction carpet to improve traction and minimize slip. The World Coordinate System, $\mathcal{W}$, and the saggital plane, $\mathcal{S}$, are shown for reference and they will be discussed in detail in Section 3. The green blow up of the robot shows the markers tracked by the Vicon system.

Accurate body tracking requires sufficient number of cameras to capture the robot. This condition imposes a constraint on the Vicon workspace that can produce viable experimental data. This smaller domain in the center of the Vicon workspace is termed the permissible domain, $\mathcal{P}$. A second data integrity issue stems from the standard Vicon calibration process which un- 
avoidably leads to a mismatch between the Vicon Coordinate System, $\mathcal{V}$, and the World Coordinate System, $\mathcal{W}$. Section 3 will outline a process to clean raw Vicon data.

For each gait in Table 1 we conducted multiple runs. We select valid stance data from only those runs where the robot achieved steady-state behavior moving straight through the permissible area, $\mathcal{P}$, along a straight path. Table 2 reports the number of conducted runs and the number of valid single tripod stance captured for each gait. Due to the higher speeds achieved by running and jogging gaits combined with the limited size of the permissible area, $\mathcal{P}$, fewer stance intervals are captured per run for these gaits in comparison to the others.

Table 2 Total and Valid Number of Runs Conducted and Stances Captured for Each Test Gait.

\begin{tabular}{lcccc}
\hline \hline & Running & Jogging & Pace & Walk \\
& (Total:Valid) & (Total:Valid) & (Total:Valid) & (Total:Valid) \\
\hline Runs & $172: 122$ & $104: 91$ & $58: 57$ & $59: 59$ \\
\hline Stances & $775: 460$ & $659: 655$ & $645: 642$ & $753: 753$ \\
\hline \hline
\end{tabular}

Each experiment run begins by starting the data logger. The robot is manually released from the distant end of the Vicon workspace with an initial speed that is roughly equal to the steady-state speed of the gait being tested in order to reduce the extend of the transient behavior. When the robot reaches the end of the workspace, first the robot and then the data logger are stopped. The raw data consisting of the proprioceptive sensor readings from the robot and the ground truth body configuration measured by the Vicon system are saved in a MAT-file. Section 3 outlines the data clean-up and extraction post-processes applied to the raw data files in preparation to the system identification study described in Section 4.

\section{Data Preparation}

The analysis presented in Section 4 and 5 concerns saggital plane behavior of the robot during steady-state locomotion without turning ${ }^{2}$. This section introduces a three step post-processing pipeline that to clean the raw data for the analysis in Section 4.

The raw data files produced by the procedure described in Section 2 contain data from two separate sources: the robot; and the Vicon system. Variables in these two data streams were sampled at different times due to the

${ }^{2}$ We also assume that there is no foot slip which is confirmed to be the case by external observations. 
difference in respective sampling frequencies and the collection start time. The first step in data preparation is the synchronization of the time stamps of these two data streams. The three computers involved in the experimental setup are on a local network isolated from the outside. The bandwidth of this network is high enough to facilitate relatively fixed and deterministic communication delay between machines which is confirmed in independent tests. Therefore, the synchronization of different data stream boils down to an offset subtraction.

At the original sampling rates $(100 \mathrm{~Hz}$ for the robot and $120 \mathrm{~Hz}$ for the Vicon) the experimental data collected within a stance interval is too small for a stable system identification study ${ }^{3}$. Therefore, the second step in data preparation is interpolation of the raw data sequences. Since all logged variables are related to the mechanical operations of the robot, they are band limited by the mechanical bandwidth (roughly $10 \mathrm{~Hz}$ ) of the passive mechanical construction of the platform. We reconstruct the analog counterpart of the acquired data by fitting a cubic spline to the recorded raw discrete-time sample sequence and then resample this analog representation at a higher frequency $(1 \mathrm{KHz})$.

The Vicon system and its shortcomings, discussed in Section 2, renders the body configuration measurements in the original Vicon Coordinate System, $\mathbf{c}^{\mathcal{V}}:=\left[\begin{array}{lllll}b_{x} & b_{y} & b_{z} & \beta & \alpha\end{array}\right]^{T} \in \mathcal{V}:=\mathbb{R}^{3} \times S O(3)$, badly unappealing for our analysis in Section 4. We will present a three step process that corrects for major sources of noise and error.

As the raw data is not sufficiently accurate outside the permissible domain, $\mathcal{P} \subset \mathcal{V}$-an experimentally identified conservative subset of the observable Vicon volume where the tracking accuracy is sufficiently high — we discard all data that falls outside this region. We also discard those runs where the robot does not follow a straight path. Those runs that produce non-null data sets are defined as "valid." The total and valid runs for each test gait is reported in Table 2.

For each run we define a Run-Specific World Coordinate System ${ }^{4}, \mathcal{W}:=$ $\mathbb{R}^{3} \times S O(3)$, as depicted in Figure 4, satisfying three conditions: 1) the horizontal plane (xy-plane) of the World frame coincides with the ground plane, $\mathcal{G}$; 2) the origin of the World frame is located where the COM trajectory projected onto the horizontal plane enters the permissible domain, $\mathcal{P}$; and 3 ) the $\mathrm{x}$-axis of the World frame is aligned with the average horizontal plane trajectory of the body COM within the permissible Vicon area. For each run we derive the coordinate transformation between the Vicon and World coordinates, $\mathbf{h}_{\mathcal{V}}^{\mathcal{W}}: \mathcal{V} \rightarrow \mathcal{W}$, in a data driven manner. Body configuration represented in the (run-specific) World Coordinate System, $\mathbf{c}^{\mathcal{W}}=\mathbf{h}_{\mathcal{V}}^{\mathcal{W}}\left(\mathbf{c}^{\mathcal{V}}\right)$, offers a simple access to the sagittal plane component of the observed COM behavior in relation to the physical ground given by $\left[b_{x} b_{z}\right]^{T}=\mathbf{P c}^{\mathcal{W}} \in \mathcal{S}$.

\footnotetext{
3 Note that the running gait stance interval is $100 \mathrm{msec}$ which only yields 10 samples at the original sampling rate.

${ }^{4}$ For the sake of simplicity this will be referred as the World Coordinates henceforth.
} 
(a)

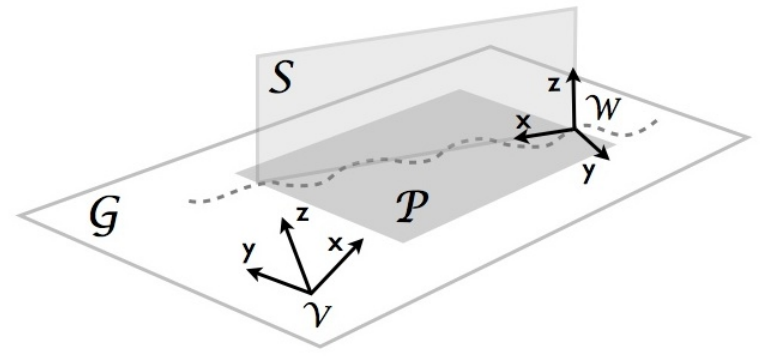

(b)

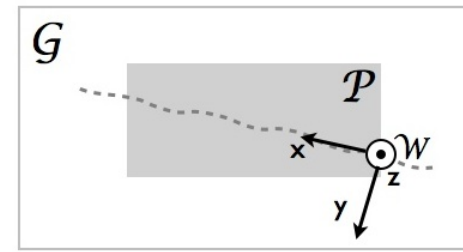

(c)

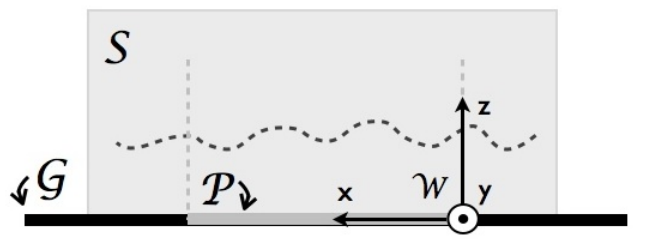

Fig. 4 Relationship between various planes and coordinate systems defined in the postprocessing operations as viewed (a) in perspective, (b) from top and (c) in sagittal view. The raw Vicon readings are represented in the Vicon Coordinate System, $\mathcal{V}$, which is not properly aligned with the movement of the robot. Its horizontal plane (xy-plane) does not coincide with the ground surface, $\mathcal{G}$. Nor any of its axes is aligned with the body trajectory. To facilitate the analysis we define the World Coordinate System, $\mathcal{W}$, whose horizontal plane (xy-plane) lays on the ground surface, $\mathcal{G}$; with its x-axis is aligned along the best line fit to the horizontal plane projection of the COM trajectory. The origin of the World Coordinate System is located at where the COM trajectory enters the permissible domain, $\mathcal{P}$.

The first derivatives of the sagittal COM configuration variables are computed by fitting a cubic spline on the available data and sampling the algebraically differentiated cubic spline. This results in the full sagittal COM state of the robot, $\left[\begin{array}{llll}b_{x} & \dot{b_{x}} & b_{z} & \dot{b_{z}}\end{array}\right]^{T}$.

The robot platform operating in a tripod gait has three basic modes ${ }^{5}$ : single tripod support; double tripod support; and aerial. The system identification study in Section 4 concerns the single tripod ground contact dynamics. This section will describe a procedure that will (approximately) extract stance

${ }^{5}$ For the sake of simplicity we are ignoring the modes where the body is supported by other combinations of legs. These operating regimes occur infrequently and are very short-lived under the steady-state conditions that our setup is designed to study. 
intervals - time intervals where the robot's body is supported and propelled by the legs of a single tripod.

(a)

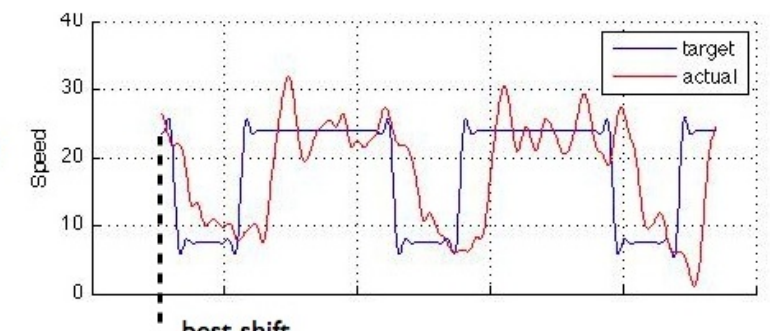

(b)

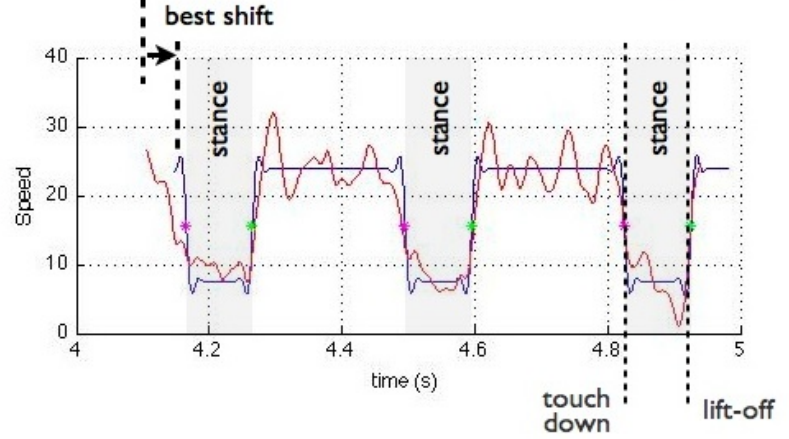

Fig. 5 (a) The averaged actual (red), $\dot{\phi_{a}}$, and target hip speed (blue), $\dot{\phi_{r}}$, for a tripod. (b) Superimposed best time shifted target speed (blue) and the averaged actual hip speed (red). The best time shift is where the cross-corrolation between target and actual speed signals is maximum. The touchdown and lift-off events are defined by the dropping and rising edges of the (best fit) shifted target speed.

The task level open-loop tripod gait [2] feeds time-parametrized two-speed target trajectories (Figure 2(b)) to hip PD position controllers. In the ideal steady-state operations a leg is in ground contact during the slow swing phase $^{6}$ of the target trajectory. Therefore, the slow swing period can serve as a conservative approximation to the stance interval. However, we note that the actual hip configuration lags that of the target while maintaining the basic form as shown in Figure 5(a). We posit that a more accurate identification of the actual physical stance interval must rely on data from the hip motor itself.

Our stance extraction process starts with the computation of the time shift,

\footnotetext{
${ }^{6}$ By design, the clock transition from fast (putative swing) to slow (putative stance) phase leads the actual ground contact event [47], however, this overlap has a very small duration relative to the full stance phase, and we make no attempt to model nor capture its effects in the system identification work reported here, relying solely upon the surrogate measures of touchdown and liftoff as described in the text.
} 


$$
t_{s}=\underset{\tau>0}{\arg \min }\left[\int\left|\dot{\phi}_{a}(t)-\dot{\phi}_{r}(t-\tau)\right|\right],
$$

that minimizes the error between the averaged actual hip speed of those hips participating in a tripod ${ }^{7}, \dot{\phi}_{a}(t):=\frac{1}{3} \sum_{i \in R \mid L}\left(\dot{\phi}_{a}^{i}(t)\right)$, and the shifted associated target speed, $\dot{\phi}_{r}\left(t-t_{s}\right)$. The shifted target speed, $\dot{\phi}_{r}\left(t-t_{s}\right)$, is a pulse train whose falling and rising edges define the touchdown and liftoff events, respectively. It directly follows that a stance interval is the time between consecutive touchdown and lift-off events. '

\section{System Identification}

Inspired by previous studies and to built on their results this work also adopts the SLIP template [39] - a point mass riding on a massless and lossless compliant leg that is attached to the body and ground surface at free rotating joints - to describe the stance mode dynamics. Note that the variation in body pitch, $\alpha$, as well as any movement outside the sagittal plane, which are unavoidable and crucial aspects of tripod behavior [1,37], are ignored for the sake of modeling simplicity. We are also ignoring aerial (ballistic flight) and double support modes of operations in our study concentrating only on the single tripod support dynamics where the body is propelled by the legs of a single tripod.

Figure 6 depicts the SLIP model employed in our study. This system is characterized by three parameters: 1) leg stiffness, $k$ in $\mathrm{N} / \mathrm{m}$; 2) leg rest length, $l_{0}$ in meters; and 3 ) fore/aft foot hold position, $f_{x}$ in meters. The body mass, $m=3.31 \mathrm{in} \mathrm{kg}$, is a constant equal to the measured robot weight. The SLIP dynamics,

$$
\ddot{\mathbf{b}}=\left[\begin{array}{c}
\ddot{b_{x}} \\
\ddot{b_{z}}
\end{array}\right]=\underbrace{-\frac{k}{m}\left(\|\mathbf{b}-\mathbf{f}\|-l_{0}\right) \frac{\mathbf{b}-\mathbf{f}}{\|\mathbf{b}-\mathbf{f}\|}}_{\mathbf{F}_{s} / m}-\mathbf{g},
$$

is driven by two forces: 1) the gravitational pull, $\mathbf{F}_{g}=m \mathbf{g}$; and 2) the spring force, $\mathbf{F}_{s}$. We define the full SLIP state vector, $\mathbf{x}:=\left[b_{x}, \dot{b_{x}}, b_{z}, \dot{b_{z}}\right]$, for the upcoming derivation and analysis discussions.

\footnotetext{
7 The process is identical for the two tripods. The reader should not that the average hip speed computation uses the proper hips depending on the tripod of interest. For notational simplicity we do not indicate the tripod explicitly in our notation.
} 


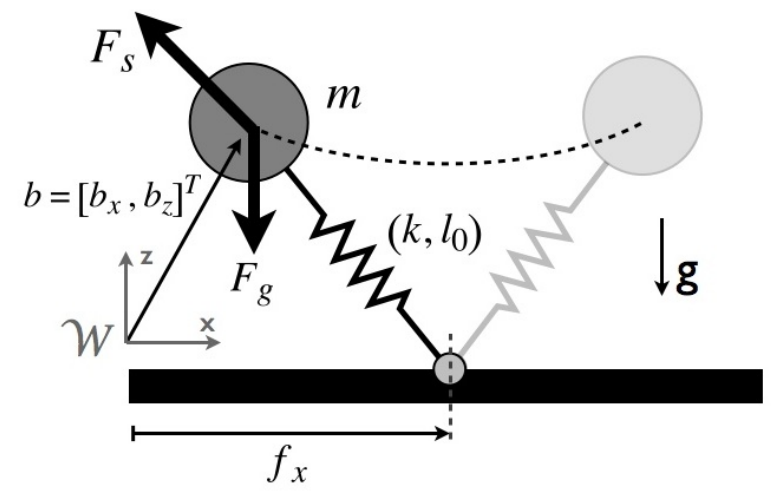

Fig. 6 SLIP model is proposed as a template model for the single tripod support dynamics of the robot. The model is parameterized by the leg stiffness, $k$, leg rest length, $l_{0}$, and the fore/aft position of the foot hold, $f_{x}$. During ground contact foot forms a free rotating joint on the ground surface, $f_{z}=0$.

For each stance interval we compute the best SLIP parameters, $\left(k, l_{0}, f_{x}\right)$, that give rise to SLIP model state trajectory ${ }^{8}$ that is the most similar to that of the given experimental data as measured by the normalized state error function,

$$
\operatorname{nerr}\left(k, l_{0}, f_{x}\right):=\frac{\sum_{i=1}^{N}\left\|\mathbf{x}_{i}^{m}\left(k, l_{0}, f_{x}\right)-\mathbf{x}_{i}^{e}\right\|}{\sum_{i=1}^{N}\left\|\mathbf{x}_{i}^{e}\right\|}
$$

where $\mathbf{x}_{i}^{e}$ is the experimentally obtained sagittal plane states of the robot COM, and $\mathbf{x}_{i}^{m}\left(k, l_{0}, f_{x}\right)$ is the model generated SLIP states for the same time instant, $i$. This error function compares the two flows across all four dimensions (positions and speeds) along the stance interval taking into account the time parameterization of the flows. The error is normalized with respect to the experimental flow and therefore represents a percentage deviation of the model away from the physical system itself.

The parameter fitting is done separately for each stance interval. Representing the data set for a stance interval by $\mathbf{X}:=\left\{\mathbf{x}_{i}^{e} \mid i \in\{1, \ldots, N\}\right\}=\mathbb{R}^{4 N}$ we define the SLIP-measure for this stance, $\sigma: \mathbb{R}^{4 N} \rightarrow \mathbb{R}$,

$$
\sigma(\mathbf{X}):=\min _{k>0, l_{0}>0, f_{x}>0}\left[\operatorname{nerr}\left(k, l_{0}, f_{x}\right)\right] .
$$

To compute SLIP-measure, $\sigma$, Nelder-Mead [48] simplex function minimization procedure is employed to minimize the normalized state error function, nerr, as a function of the SLIP parameters, $\left(k, l_{0}, f_{x}\right)$, whose result,

\footnotetext{
8 Model trajectory evaluation starts from the same initial conditions as the given experimental data. We employed MATLAB ode45 numerical integration tool for our computations.
} 


$$
\left(k^{*}, l_{0}^{*}, f_{x}^{*}\right)=s(\mathbf{X}):=\underset{k>0, l_{0}>0, f_{x}>0}{\arg \min }\left[\operatorname{nerr}\left(k, l_{0}, f_{x}\right)\right]
$$

provides the parameterization of the best fitting SLIP model for the stance interval data at hand.

We start the minimization process ${ }^{9}$ from an initial guess, $\left(k^{0}, l_{0}^{0}, f_{x}^{0}\right)=$ $\left[2000.0,0.15, \operatorname{mid}\left(b_{x}^{e}\right)\right]$, where $\operatorname{mid}\left(b_{x}^{e}\right)$ is the middle of the horizontal interval swept by the experimental data being fitted.

\section{Energetics and Dynamic Behaviors}

We measure the energetic efficiency of locomotion by specific resistance [4]a widely used dimensionless measure of performance introduced to robotics in [11] that is normalized against the weight of the locomotor,

$$
\mathrm{SR}:=\frac{\bar{P}}{m g \bar{v}}
$$

where $\bar{P}$ is the average power consumption and $\bar{v}$ is the average speed. Specific resistance,SR, average power, $\bar{P}$, and average speed, $\bar{v}$, are all computed on a per run basis and they are associated with all valid stance intervals captured in that run.

Since the platform remains unchanged in our study the average power, $\bar{P}$, and speed, $\bar{v}$, are the only two variables that affect the specific resistance, SR. Figure 7 and 8 are scatter plots illustrating the distribution of the three energetic performance measures. We observe a very clear clustering for each test gait. Table 3 reports the mean and standard deviations of the energetic performance measures for each test gait.

Table 3 The Average Energetic Parameters for the Test Gaits

\begin{tabular}{|l|cc|cc|cc|}
\hline \hline & \multicolumn{2}{|c|}{ nerr } & \multicolumn{2}{|c|}{$\bar{P}(\mathrm{~W})$} & \multicolumn{2}{c|}{$\bar{v}(\mathrm{~m} / \mathrm{s})$} \\
& mean & std & mean & std & mean & std \\
\hline Running & 0.087 & 0.07 & 31.63 & 2.99 & 1.06 & 0.04 \\
\hline Jogging & 0.26 & 0.10 & 42.92 & 1.43 & 0.64 & 0.01 \\
\hline Pace & 0.52 & 0.11 & 26.23 & 0.51 & 0.30 & 0.004 \\
\hline Walk & 0.54 & 0.03 & 20.93 & 0.32 & 0.16 & 0.002 \\
\hline
\end{tabular}

We observe a clear monotonic relationship between the specific resistance, $\mathrm{SR}$, and the average speed, $\bar{v}$, in Figure 7 which is not unexpected [16].

9 Each SLIP parameter is defined over a different range of values. While relevant leg stiffness, $k$, values are in thousands, same for the rest length, $l_{0}$, is less then one. In order to avoid near singular search simplexes in the Nelder-Mead iterations the three SLIP parameters are normalized. 
However, the variation of the specific resistance, SR, is not driven by the difference in speed. Figure 8 shows that the the running gait is not only faster than the jogging gait but also consumes less power. This is a very important observation which will be revisited later in this section.
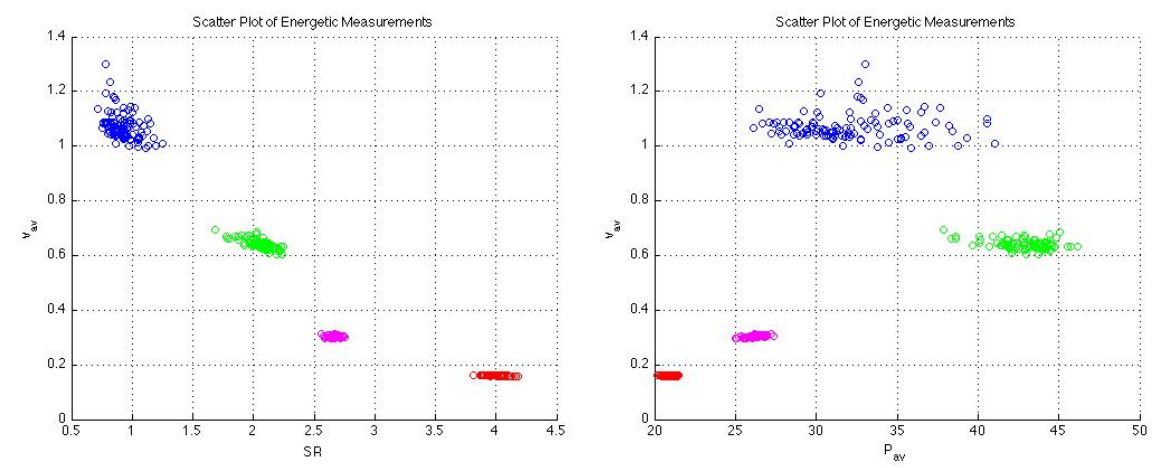

Fig. 7 The scatter plot of average speed, Fig. 8 The scatter plot of average power, $\bar{P}$, $\bar{v}$, versus specific resistance, SR, for all valid versus average speed, $\bar{v}$, for all valid stance stance intervals captured in the entire exper- intervals captured in the entire experimenimental data. Each test gait is color coded: tal data. Each test gait is color coded: runrunning (blue); jogging (green); pace (pur- ning (blue); jogging (green); pace (purple); ple); and walk (red). and walk (red). We notice that the running gait consumes less power even though it has a higher average speed compared to the jogging gait.

In our analysis we choose to measure the quality of dynamical activity in a gait by its similarity to what can be produced by a SLIP model. The SLIPmeasure, $\sigma$, in Equation 3 gauges the best case deviation between the state flows of the physical system and the SLIP model starting at the same initial condition. It simply follows that SLIP-measure, $\sigma$, offers an effective measure of the quality of dynamical activity associated with a gait.

Figure 9 demonstrates the distribution of the SLIP-measure, $\sigma$, and the specific resistance, $\mathrm{SR}$, for each stance. The plot suggests a monotonic relationship between the two measures supporting the hypothesis that gaits exhibiting a more pronounced degree this particular quality of dynamical activity realize better energetic efficiency than gaits exhibiting less.

\section{Conclusion and Future Work}

In this paper we describe an experimental setup to collect large quantities of empirical data from an untethered dynamic legged robotic platform. Four representative locomotion gaits with varying dynamical characteristics are 




Fig. 9 The scatter plot of SLIP-measure, $\sigma$, (normalized state error evaluated at the fitted parameters) versus specific resistance, SR, for all valid stance intervals captured in the entire experimental data. Each test gait is color coded: running (blue); jogging (green); pace (purple); and walk (red). The normalized error is a measure of level of dynamism in a gait. We observe a clear monotonic relationship between the dynamism and energetic efficiency of gaits.

studied. We outline a post-processing procedure that cleans the raw data and effectively extracts single tripod support intervals during steady-state tripod gait locomotion. Our procedure is founded on a proprioception based (using joint space measurements) stance detection mechanism.

Biological studies [4-7] suggest that the ability to effectively recirculate mechanical energy through spring-mass mechanics confers significant energetic benefits in animals, and that fast biological runners adopt the specific mechanics of the SLIP [28]. RHex-class robots can capture this aspect of animal locomotion and demonstrate energetically efficient locomotion as they can passively embed the SLIP dynamics in carefully tuned open-loop controlled gaits [40]. Results presented in this paper further supports this early discovery with a richer data set.

Our long term goal is to develop a unified constructive approach to gait design where both the mechanical [44] and algorithmic aspects are considered in an integrated manner. Notwithstanding a decade's effort [35,49-51], mathematical analysis that can account for the emergence of SLIP dynamics in RHex-style systems has proven challenging. Even the narrower analysis relating open-loop RHex clock control parameter settings to effective SLIP locomotion outcomes has proven mathematically difficult [49]. Moreover, it is not clear that the more tractable active sensor-based closed-loop methods [41,52] 
for anchoring SLIP dynamics in RHex will yield energetic benefits comparable to their open-loop counterparts. Consequently, empirical tuning [22] remains the chief means of achieving high quality gaits in RHex-class platforms at this time.

Our results in this paper display a strong correlation between a gait's degree of dynamical exchange, as measured by the SLIP-measure, $\sigma$, and its energetic efficiency, as measured by the specific resistance, SR, [4]. The data also show that dynamic behaviors achieve higher locomotion efficiency not simply through increased speed but also through decreased power consumptionsuggesting that a more efficient recruitment of total system energy might be achieved via more SLIP like dynamics. These new results encourage the shift to a potentially more tractable rational design paradigm wherein the simpler SLIP mechanics itself becomes the explicit target of control.

This empirical relationship between the SLIP-measure, $\sigma$, and energetic efficiency, SR, reported in this paper suggests that tuning studies focusing on energetic performance can employ the SLIP-measure, $\sigma$, as an alternative to the specific resistance, SR. One immediate use for the gait qualification associated with SLIP-measure, $\sigma$, is the ability to impose functional optimization constraints that are informed by the mathematical properties of the SLIP template and desired gait characteristics (e.g. walking is characterized by stiff leg and short strides). We expect that such an approach would help reduce the uncertainty in the optimization process that aims to produce a particular gait style (walking versus running while optimizing energetics) and ultimately reduce the required number of experiments.

The next paper in this series will investigate constrained SLIP-measure based gait tuning. At this time it is not clear how that recourse would represent a substantial improvement over present practice. Work now in progress explores empirically the performance gains associated with such a constrained SLIP-measure based approach to gait tuning. More broadly, the results we report here represent yet another significant motivation for pushing ahead with the mathematical investigation of how to anchor [39] SLIP in RHex.

Acknowledgements This work is supported in part by the National Science Foundation (NSF) under a FIBR Award 0425878. Yasemin Ozkan Aydin is supported by International Research Fellowship Programme of The Scientific and Technological Research Council of Turkey (TUBITAK).

\section{References}

1. S. Burden, J. E. Clark, J. D. Weingarten, H. Komsuoglu, and D. E. Koditschek, "Heterogeneous leg stiffness and roll in dynamic running," in Proceedings of IEEE Conference of Robotics and Automation, 2007.

2. U. Saranli, M. Buehler, and D. E. Koditschek, "Rhex - a simple and highly mobile hexapod robot," International Journal of Robotics Research, vol. 20, no. 7, pp. 616- 
$631,2001$.

3. R. Blickhan, "The spring-mass model for running and hopping," Journal of Biomechanics, vol. 22, no. 11/12, pp. 1217-1227, 1989.

4. G. Gabrielli and T. v. Karman, "What price speed?" Mechanical Engineering, pp. 775-781, 1950.

5. C. H. Greenewalt, "The energetics of locomotion-is small size really disadvantageous?" Proceedings of the American Philosophical Society, vol. 121, pp. 100-106, 1976.

6. G. A. Cavagna, N. C. Heglund, and C. R. Taylor, "Mechanical work in terrestrial locomotion: two basic mechanisms for minimizing energy expenditure," American Journal of Physiology, vol. 5, no. 233, pp. R243-R261, 1977.

7. C. R. Taylor, N. C. Heglund, and G. M. O. Maloiy, "Energetics and mechanics of terrestrial locomotion," Journal of Experimental Biology, vol. 97, pp. 1-21, 1982.

8. T. J. Roberts, R. Kram, P. G. Weyand, and C. R. Taylor, "Energetics of bipedal running," The Journal of Experimental Biology, vol. 201, pp. 2745-2751, 1998.

9. A. E. Kerdok, A. A. Biewener, T. A. McMahon, P. G. Weyand, and H. M. Herr, "Energetics and mechanics of human running on surfaces of different stiffnesses," Journal of Applied Physiology, vol. 92, p. 469478, 2002.

10. H. M. Herr, G. T. Huang, and T. A. McMahon, "A model of scale effects in mammalian quadrupedal running," Journal of Experimental Biology, vol. 205, pp. 959-967, 2002.

11. P. Gregorio, M. Ahmadi, and M. Buehler, "Design, control, and energetics of an electrically actuated legged robot," Systems, Man, and Cybernetics, Part B: Cybernetics, IEEE Transactions on, vol. 27, no. 4, pp. 626-634, Aug 1997.

12. H. B. Brown, Jr. and G. Zeglin, "The bow leg hopping robot," in Proceedings of International Conference on Robotics and Automation, 1998.

13. M. Ahmadi and M. Buehler, "The arl monopod ii running robot: Control and energetics," in International Conference on Robotics and Automation, 1999.

14. M. Garcia, A. Chatterjee, and A. Ruina, "Efficiency, speed, and scaling of twodimensional passive-dynamic walking," Dynamics and Stability of Systems, vol. 15, no. 2, pp. 75-99, 2000.

15. J. D. Weingarten, G. A. D. Lopes, M. Buehler, G. R. E., and D. E. Koditschek, "Automated gait adaptation for legged robots," in Int. Conf. Robotics and Automation. New Orleans, USA: IEEE, 2004.

16. R. J. Full, "Concepts of efficiency and economy in land locomotion," in Efficiency and Economy in Animal Physiology, R. W. Blake, Ed. Cambridge University Press, 1991, pp. $97-131$.

17. M. B. Binnard, "Design of a small pneumatic walking robot," MS, Massachusetts Institute of Technology, 1995.

18. G. Zeglin, "The bow leg hopping robot," Doctoral Thesis in Robotics, Carnegie Mellon University, 1999.

19. D. McMordie and M. Buehler, "Towards pronking with a hexapod robot," in Proceedings of 4 th International Conference on Climbing and Walking Robots, Germany, Sep. 2001.

20. D. Campbell and M. Buehler, "Preliminary bounding experiments in a dynamic hexapod," in Experimental Robotics, B. Siciliano and P. Dario, Eds. Springer-Verlag, 2003, ch. VIII, pp. 612-621.

21. K. Autumn, M. Buehler, M. Cutkosky, R. S. Fearing, R. J. Full, D. I. Goldman, R. Groff, W. Provancher, A. A. Rizzi, U. Saranli, A. Saunders, and D. E. Koditschek, "Robotics in scansorial environments," in Proceedings of SPIE 2005, 2005, pp. 291302.

22. D. E. Koditschek, R. J. Full, and B. M., "Mechanical aspects of legged locomotion control," Antropod Structure and Development, vol. 33, pp. 251-272, 2004.

23. R. F. Ker, M. B. Bennett, S. R. Bibby, R. C. Kester, and R. M. Alexander, "The spring in the arch of the human foot," Nature, vol. 325, pp. 147-149, 1987.

24. R. M. Alexander and H. C. Bennet-Clark, "Storage of elastic strain energy in muscle and other tissues," Nature, vol. 265, pp. 114-117, Jan. 1977. 
25. R. M. Alexander, "Three uses for springs in legged locomotion," The International Journal of Robotics Research, vol. 9, no. 2, pp. 53-61, 1990.

26. Alexander, R. McN., Elastic mechanisms in animal movement. Cambridge University Press, 1988.

27. M. H. Dickinson, C. T. Farley, R. J. Full, M. A. R. Koehl, R. Kram, and S. Lehman, "How animals move: An integrative view," Science, vol. 288, pp. 100-106, Apr. 2000.

28. R. Blickhan and R. J. Full, "Similarity in multilegged locomotion: Bouncing like a monopode," Journal of Comparative Physiology, vol. 173, pp. 509-517, 1993.

29. H. Komsuoglu, "Dynamic legged mobility—an overview," in Proceedings of International Joint Robotics Conference and Workshop, 2009.

30. M. H. Raibert, Legged robots that balance, ser. MIT Press series in artificial intelligence. Boston: MIT Press, 1986.

31. M. D. Berkemeier and K. V. Desai, "Design of a robot leg with elastic energy storage, comparison to biology, and preliminary experimental results," in Proceedings of IEEE Conference on Robotics and Automation, vol. 1, Minneapolis, Apr. 1996, pp. 213-218.

32. M. Buehler, R. Battaglia, A. Cocosco, G. Hawker, J. Sarkis, and K. Yamazaki, "Scout: A simple quadruped that walks, climbs, and runs," in International Conference on Robotics and Automation, 1998.

33. B. McBride, R. Longoria, and E. Krotkov, "Off-road mobility of small robotic ground vehicles," Measuring the Performance and Intelligence of Systems: Proceedings of the 2003 PerMIS Workshop, vol. NIST Special Publication 1014, pp. 405-412, September 16-18 2003, edited by Messina, E. and Meystel, A.

34. C. Georgiades, A. Hogue, H. Liu, A. Ripsman, R. Sim, L. A. Torres, P. Zhang, C. Prahacs, M. Buehler, G. Dudek, M. Jenkin, and E. Milios, "Aqua: an aquatic walking robot," Dalhousie University, Technical Report CS-2003-08, Nov. 2003.

35. U. Saranli, A. Rizzi, and D. E. Koditschek, "Model-based dynamic self-righting maneuvers for a hexapedal robot," International Journal of Robotics Research, vol. 23, no. 9, pp. 903-918, Sep. 2004.

36. J. C. Spagna, D. I. Goldman, P.-C. Lin, D. E. Koditschek, and R. J. Full, "Distributed feet enhance mobility in many-legged animals and robots," Journal of Bioinspiration and Biomimetics, vol. 2, no. 1, pp. 9-18, March 2007.

37. H. Komsuoglu, K. Sohn, R. J. Full, and D. E. Koditschek, "A physical model for dynamical arthropod running on level ground," in Proceedings of 11th International Symposium on Experimental Robotics, 2008.

38. C. Li, P. B. Umbanhowar, H. Komsuoglu, D. E. Koditschek, and D. I. Goldman, "Sensitive dependence of the motion of a legged robot on granular media," Proceedings of National Academy of Science (PNAS), vol. 106, no. 9, pp. 3029-3034, Feb. 2009. [Online]. Available: http://www.pnas.org/content/106/9/3029.full.pdf+html

39. R. J. Full and D. E. Koditschek, "Templates and anchors: Neuromechanical hypotheses of legged locomotion," The Journal of Experimental Biology, vol. 202, no. 23, pp. 33253332, 1999.

40. R. Altendorfer, U. Saranli, H. Komsuoglu, D. E. Koditschek, H. B. Brown, Jr., M. Buehler, N. Moore, D. McMordie, and R. J. Full, "Evidence for spring loaded inverted pendulum running in a hexapod robot," in Proceedings on International Symposium on Experimental Robotics, 2000.

41. U. Saranli and D. E. Koditschek, "Template based control of hexapedal running," in Proceedings of International Conference on Robotics and Automation, vol. 1, Sep. 2003, pp. 1374-1379.

42. R. M. Alexander and A. S. Jayes, "Vertical movements in walking and running," Journal of Zoology, vol. 185, pp. 27-40, 1978.

43. Alexander, R. McN. and Jayes, A. S., "Fourier analysis of forces exerted in walking and running," Journal of Biomechanics, vol. 13, pp. 383-390, 1980.

44. K. C. Galloway, J.E.Clark, and D. E. Koditschek, "Design of a tunable stiffness composite leg for dynamic locomotion," In Proceedings of the ASME Int.Design Engineering Tech. Conferences, 2009. 
45. H. Komsuoglu and D. Mellinger, "Surface classification with a dynamic hexapod robot," in Proceedings of international Symposium on Experimental Robotics, 2010, in preparation.

46. J. D. Weingarten, D. E. Koditschek, H. Komsuoglu, and C. Massey, "Robotics as the delivery vehicle: A contexualized, social, self paced, engineering education for life-long learners," in Proceedings of Robotics and System Science Conference, 2007.

47. H. Komsuoglu, D. McMordie, U. Saranli, N. Moore, M. Buehler, and D. E. Koditschek, "Proprioception based behavioral advances in a hexapod robot," in Proceedings of International Conference on Robotics and Automation, Seoul, Korea, 2001.

48. J. A. Nelder and R. Mead, "A simplex method for function minimization," Computer Journal, vol. 7, no. 4, pp. 308-313, 1965. [Online]. Available: http://comjnl.oxfordjournals.org/cgi/content/abstract/7/4/308

49. R. Altendorfer, D. E. Koditschek, and P. J. Holmes, "Stability analysis of a clockdriven rigid-body slip model of rhex," International Journal of Robotics Research, vol. 23, no. 10-11, pp. 1001-1012, 2004.

50. P. J. Holmes, D. E. Koditschek, R. J. Full, and J. Guckenheimer, "Dynamics of legged locomotion: Models, analysis and challenges," Society of Industrial and Applied Mathematics, vol. 48, no. 2, pp. 207-304, 2006. [Online]. Available: http://repository.upenn.edu/ese_papers/200/

51. J. E. Seipel and P. J. Holmes, "A simple model for clock-actuated legged locomotion," Journal of Regular and Chaotic Dynamics, vol. 12, no. 5, pp. 502-520, 2007.

52. U. Saranli, "Dynamic locomotion in a hexapod robot," $\mathrm{PhD}$, Univerisity of Michigan, 2002.

53. A. Johnson, G. C. Haynes, and D. E. Koditschek, "Disturbance detection, identication, and recovery by gait transition in legged robots," in Proceedings of IEEE/RSJ International Conference on Intelligent Robots and Systems, 2010, submitted. 